\title{
NEW VASCULAR PLANT FLORISTIC RECORDS FOR THE DAKOTAS
}

\author{
Karen Meyer Walker ${ }^{1}$, Ashley Pemberton ${ }^{2}$, Tyler Grassrope ${ }^{3}$, Kayla Gravatt ${ }^{3}$, \\ Sunshine Claymore ${ }^{4}$, and Wendy L. Applequist ${ }^{1,5}$
}

\begin{abstract}
Recent collections of vascular plants on the Crow Creek Indian Reservation in central South Dakota include 64 new county records for Hughes County, 72 for Buffalo County, and 1 for Hyde County. Additionally, 2 new county records are reported for Sioux County in southern North Dakota.
\end{abstract}

Resumen.-Las recientes colectas de plantas vasculares en la Reserva Indie Crow Creek en el centro de Dakota del Sur incluyen: 64 nuevos registros en el Condado Hughes, 72 en el Condado Buffalo y uno en el Condado Hyde. Además, se reportaron dos nuevos registros en el Condado Sioux, en el sur de Dakota del Norte.

The Crow Creek Indian Reservation encompasses portions of Hughes, Hyde, and Buffalo counties along the east side of the Missouri River in central South Dakota. Botanical collections were made on the reservation as part of an effort to compile a list of its flora. These collections were identified and compared to available reports of plant species distributions in South Dakota, including those from the Atlas of the Flora of the Great Plains (Great Plains Flora Association 1977), the USDA Plants Database (USDA-NRCS 2013), and the Biota of North America Program (BONAP) website (Kartesz 2013). Distributions given in Flora of the Great Plains (Great Plains Flora Association 1991) seldom mention specific counties, and few county records appear to have been published from this area since the publication of the Great Plains Flora Association reference volumes.

A surprisingly large number of species, including widespread and well-known species, were identified as being new county records according to the available literature and databases. This seems to be in part because the entire central portion of South Dakota is badly undercollected. Card et al. (2010), while databasing known herbarium specimens from counties west of the Missouri River, reported collection numbers of $0.02-0.08$ specimens $\cdot \mathrm{km}^{-2}$ for the nearby Dewey, Lyman, and Stanley counties, as compared to 1.07-8.73 specimens $\cdot \mathrm{km}^{-2}$ in well-collected southwestern counties. At the extreme, they reported locating only 97 specimens from the entirety of Dewey Co. It seems certain in the most neglected counties that many species have not only not been databased but have never been collected. However, the broader distributional ranges given for some species in this region by BONAP, as compared to the Atlas, suggest that many county records may have have been collected in recent decades but not published as such.

Therefore, we herein report new county records in South Dakota for 123 species. Additionally, some collections were made for a project in southern North Dakota, from which 2 new records in Sioux County are reported. Common names and map numbers, where given, are from the Atlas of the Flora of the Great Plains (Great Plains Flora Association 1977), and the name used in that reference, if not the currently preferred name, is given in brackets, following the useful format that Rolfsmeier et al. (1988) employed in reporting records from Nebraska. Family assignments have been updated to correspond to the Angiosperm Phylogeny Group III (APG III) classification (Angiosperm Phylogeny Group 2009). Voucher specimens are preserved in the Missouri Botanical Garden herbarium (MO) and at the Crow Creek tribal high school. Most coordinates given were obtained by GPS at the time of collection; some, given only to the minute, were estimated from maps.

\footnotetext{
${ }^{1}$ Missouri Botanical Garden, Box 299, St. Louis, MO 63166-0299.

2 Maryville University, 650 Maryville University Dr., St. Louis, MO 63141.

${ }^{3}$ Crow Creek High School, 101 Crow Creek Loop, Stephan, SD 57346.

${ }^{4}$ Sitting Bull College, 9299 N Dakota 24, Fort Yates, ND 58538.

5Corresponding author. E-mail: wendy.applequist@mobot.org
} 


\section{SOUTH Dakota County RECordS}

Abutilon theophrasti Medik. (Malvaceae), velvetleaf. Map 349. BUFFALO County (1.0 mile $\mathrm{S}$ on Hwy. 47 from Hwy. 34, at bridge, 44 2.9' N, $99^{\circ} 24.6^{\prime} \mathrm{W}$, Meyer \& Grassrope 1328).

Acer negundo L. (Sapindaceae), box elder. HUGHES County (Joe Creek Recreation Area, along Missouri River, $44^{\circ} 8.8^{\prime}$ N, $99^{\circ} 47.8^{\prime}$ W, Meyer 1343).

Achillea millefolium L. (Asteraceae), yarrow. Map 331. BUFFALO County (334th Ave. off 26th Ave., 1.5 mi past Log House, $44^{\circ} 8.8^{\prime} \mathrm{N}, 99^{\circ}$ $31.2^{\prime} \mathrm{W}$, Meyer et al. 1524).

Allium textile A. Nels. \& J.F. Macbr. (Amaryllidaceae), white wild onion. Map 2146. HUGHES County (Joe Creek Recreation Area, $44^{\circ} 8.7^{\prime} \mathrm{N}, 99^{\circ}$ $47.8^{\prime} \mathrm{W}$, Townesmith \& Applequist 2460; near stock dam W of Rd. $21756 \mathrm{~S}$ of Hwy. 34, 44 $15.3^{\prime} \mathrm{N}, 99^{\circ} 41.6^{\prime} \mathrm{W}$, Applequist \& Townesmith 335 ) and BUFFALO County (steep bluffs along SD route $50,2.8 \mathrm{mi}$ S of SD route $47,43^{\circ} 57.9^{\prime} \mathrm{N}$, $99^{\circ} 18.5^{\prime} \mathrm{W}$, Townesmith \& Applequist 2458; along unimproved extension of 334th Ave., 3.0 mi N of 226th St., hillsides above Missouri River, $44^{\circ} 8.8^{\prime} \mathrm{N}, 99^{\circ} 31.2^{\prime} \mathrm{W}$, Townesmith 2467).

Amaranthus hybridus L. (Amaranthaceae), slender pigweed. Map 282. This weedy species is common in the southeastern quadrant of the Great Plains, with few populations known in the northern and western portions. BUFFALO County $(2.2 \mathrm{mi}$ on Hwy. $47 \mathrm{~S}$ of Ft. Thompson, toward Missouri River, $44^{\circ} 2.9^{\prime} \mathrm{N}, 99^{\circ} 24.6^{\prime} \mathrm{W}$, Meyer \& Grassrope 1321) and HUGHES County (4 mi S on Rd. 21756 off Hwy. 34, at Missouri River, Meyer et al. 1517).

Amaranthus palmeri S. Watson (Amaranthaceae), Palmer's pigweed. Map 283. This species is usually confined to the southern half of the Great Plains, though BONAP reports it to be present in 2 counties at the western edge of South Dakota. BUFFALO County (Hwy. 47, S 2 mi from Hwy. 34, at Rd. 235, Crow Creek, 4358.6 $\mathrm{N}, 99^{\circ} 18.9^{\prime} \mathrm{W}$, Meyer \& Grassrope 1337).

Amaranthus powellii S. Watson (Amaranthaceae), Powell's pigweed. This primarily western species is widespread but only rarely reported from the Great Plains; BONAP reports it to be present in 3 counties in western South Dakota. BUFFALO County $(0.5 \mathrm{mi} \mathrm{N}$ of 236 St. \& 349 Ave., $43^{\circ} 58.0^{\prime} \mathrm{N}, 99^{\circ} 12.4^{\prime} \mathrm{W}$, Meyer et al. 1481).

Ambrosia artemisiifolia L. (Asteraceae), common ragweed. Map 1326. BUFFALO County $(0.5$ mi $\mathrm{N}$ of 236 St. \& 349 Ave., $43^{\circ} 58.0^{\prime} \mathrm{N}, 99^{\circ}$ 12.4' W, Meyer et al. 1300; Hwy. 47, 2 mi S of Hwy. 34, at Rd. 235, Crow Creek, $43^{\circ} 58.6^{\prime} \mathrm{N}$, $99^{\circ} 18.9^{\prime} \mathrm{W}$, Meyer \& Grassrope 1334).

Amorpha nana Nutt. (Fabaceae), dwarf wild indigo. Map 631. BUFFALO County (1 mi N of Ft.
Thompson on Hwy. 47, then E 0.5-1 mi, $44^{\circ} 5.2^{\prime} \mathrm{N}, 99^{\circ} 25.7^{\prime} \mathrm{W}$, Meyer \& Pemberton 1440).

Andropogon gerardii Vitman (Poaceae), big bluestem. Map 1883. BUFFALO County $(1.0 \mathrm{mi} \mathrm{S}$ on Hwy. 47 from Hwy. 34, at bridge, $44^{\circ} 2.9^{\prime} \mathrm{N}$, $99^{\circ} 24.6^{\prime} \mathrm{W}$, Meyer \& Grassrope 1330).

Anemone canadensis L. (Ranunculaceae), meadow anemone. Map 66. BUFFALO County $(0.5 \mathrm{mi} \mathrm{N}$ of 236 St. \& 349 Ave., $43^{\circ} 58.0^{\prime} \mathrm{N}, 99^{\circ} 12.4^{\prime} \mathrm{W}$, Meyer et al. 1560).

Anemone cylindrica A. Gray (Ranunculaceae), candle anemone. Map 68. BUFFALO County $(0.5 \mathrm{mi}$ $\mathrm{N}$ of 236 St. \& 349 Ave., $43^{\circ} 58.0^{\prime} \mathrm{N}, 99^{\circ} 12.4^{\prime} \mathrm{W}$, Meyer et al. 1561).

Anemone patens L. (Ranunculaceae), pasqueflower. Map 71. HUGHES County (stock dam W of Rd. $21756 \mathrm{~S}$ of Hwy. $34,44^{\circ} 15.3^{\prime} \mathrm{N}, 99^{\circ} 41.6^{\prime} \mathrm{W}$, Applequist \& Townesmith 334).

Artemisia absinthium L. (Asteraceae), wormwood. Map 1344. HUGHES County (1.3 mi off West Bend Rd. on Rd. 15/320, roadside, $44^{\circ} 9.6^{\prime} \mathrm{N}$, $99^{\circ} 46.0^{\prime} \mathrm{W}$, Meyer et al. 1486).

Artemisia dracunculus L. (Asteraceae), silky wormwood. Map 1349. HUGHES County $(2.6 \mathrm{mi} \mathrm{S}$ of Hwy. 34 on Rd. 21756, $44^{\circ} 13.6^{\prime} \mathrm{N}, 99^{\circ} 41.1^{\prime} \mathrm{W}$, Meyer et al. 1463).

Artemisia frigida Willd. (Asteraceae), prairie sagewort. Map 1351. HUGHES County (Joe Creek Recreation Area, $44^{\circ} 8.8^{\prime} \mathrm{N}, 99^{\circ} 47.8^{\prime} \mathrm{W}$, Meyer et al. 1568).

Artemisia longifolia Nutt. (Asteraceae), long-leaved sage. Map 1352. BUFFALO County $(0.5 \mathrm{mi} \mathrm{N}$ on Hwy. 37 from Ft. Thompson, then E 0.5-1 mi, $44^{\circ} 5.2^{\prime} \mathrm{N}, 99^{\circ} 25.7^{\prime} \mathrm{W}$, Meyer \& Pemberton 1446 and Meyer \& Pemberton 1447, $2.5 \mathrm{mi} \mathrm{S}$ on Hwy. 47 from Hwy. 50, 43⒌ 57.9' N, 99 18.4' W, Meyer et al. 1574).

Artemisia ludoviciana Nutt. var. ludoviciana (Asteraceae), white sage. Map 1353. BUFFALO County $(0.5 \mathrm{mi} \mathrm{N}$ of $236 \mathrm{St}$ \& 349 Ave., $43^{\circ} 58.0^{\prime} \mathrm{N}, 99^{\circ} 12.4^{\prime} \mathrm{W}$, Meyer et al. 1557).

Asclepias incarnata L. (Apocynaceae), swamp milkweed. Map 989. HUGHES County $(2.5 \mathrm{mi} \mathrm{E}$ of West Bend Rd./15 \& 320 Rd., $0.6 \mathrm{mi}$ on access road to Missouri River, $44^{\circ} 8.5^{\prime} \mathrm{N}, 99^{\circ} 49.0^{\prime} \mathrm{W}$, Meyer et al. 1497).

Asclepias speciosa Torr. (Apocynaceae), showy milkweed. Map 996. BUFFALO County $(0.5 \mathrm{mi} \mathrm{N}$ of 236 St. \& 349 Ave., $43^{\circ} 58.0^{\prime} \mathrm{N}, 9^{\circ} 12.4^{\prime} \mathrm{W}$, Meyer \& Pemberton 1417) and HUGHES County $(0.5 \mathrm{mi} \mathrm{E}$ of West Bend Rd./15 \& 320 Rd., $0.6 \mathrm{mi}$ on access road to Missouri River, $44^{\circ} 8.5^{\prime} \mathrm{N}, 99^{\circ} 49.0^{\prime} \mathrm{W}$, Meyer et al. 1505).

Asclepias viridiflora Raf. (Apocynaceae), green milkweed. Map 1003. BUFFALO County $(0.5 \mathrm{mi}$ $\mathrm{N}$ of 236 St. \& 349 Ave., $43^{\circ} 58.0^{\prime} \mathrm{N}, 99^{\circ} 12.4^{\prime} \mathrm{W}$, Meyer et al. 1477).

Astragalus agrestis G. Don (Fabaceae). Map 636. BUFFALO County (1/2 mi N of 236th St. and 
349th Ave., $43^{\circ} 58.0^{\prime} \mathrm{N}, 99^{\circ} 12.4^{\prime} \mathrm{W}$, Applequist \& Townesmith 325).

Bromus inermis Leyss. (Poaceae), smooth brome. Map 1913. BUFFALO County $(0.5 \mathrm{mi} \mathrm{N}$ of 236 St. \& 349 Ave., $43^{\circ} 58.0^{\prime} \mathrm{N}, 99^{\circ} 12.4^{\prime} \mathrm{W}$, Meyer et al. 1247).

Bromus japonicus Thunb. ex Murray (Poaceae), Japanese brome. Map 1914. BUFFALO County (0.5 mi N of 236 St. \& 349 Ave., 4358.0' N, $99^{\circ} 12.4^{\prime} \mathrm{W}$, Meyer et al. 1246) and HUGHES County (2.2 mi off Hwy. $34 \mathrm{~W}$ on Rd. 21756, $44^{\circ} 14^{\prime} \mathrm{N}, 99^{\circ} 4^{\prime} \mathrm{W}$, Meyer et al. 1537).

Calystegia sepium (L.) R. Br. subsp. angulata Brummitt [Convolvulus sepium L.] (Convolvulaceae), hedge bindweed. Map 1035. HUGHES County (2.6 mi off Hwy. $34 \mathrm{~W}$ on Rd. 21756, $44^{\circ} 14^{\prime} \mathrm{N}, 99^{\circ} 41^{\prime} \mathrm{W}$, Meyer et al. 1289).

Carex brevior (Dewey) Mack. ex Lunell (Cyperaceae), fescue sedge. Map 1739. BUFFALO County ( $1 \mathrm{mi} \mathrm{N}$ on Hwy. 47 from Ft. Thompson, then E $0.5-1 \mathrm{mi}, 44^{\circ} 5.2^{\prime} \mathrm{N}, 99^{\circ} 25.7^{\prime} \mathrm{W}$, Meyer \& Pemberton 1444).

Carex eleocharis L.H. Bailey (Cyperaceae), needleleaf sedge. Map 1754. HUGHES County (around North Bend Recreation Area, 44 $13^{\prime} 34^{\prime \prime}$ N, 99 41' 06" W, Applequist \& Townesmith 323).

Carex vulpinoidea Michx. (Cyperaceae), fox sedge. Map 1806. HUGHES County (2.6 mi S of Hwy. 34 on Rd. $21756,44^{\circ} 13.6^{\prime} \mathrm{N}, 99^{\circ} 41.1^{\prime} \mathrm{W}$, Meyer et al. 1467).

Castilleja sessiliflora Pursh (Orobanchaceae), downy paintbrush. Map 1209. BUFFALO County $(0.5$ mi N of 236 St. \& 349 Ave., $43^{\circ} 58.0^{\prime} \mathrm{N}, 99^{\circ}$ 12.4' W, Meyer et al. 1564).

Celastrus scandens L. (Celastraceae), climbing bittersweet. Map 822. HUGHES County (S from Hwy. 34 on Rd. 21756, W on side road, at stock dam, $44^{\circ} 15.3^{\prime} \mathrm{N}, 99^{\circ} 41.6^{\prime} \mathrm{W}$, Meyer \& Pemberton 1425).

Celtis occidentalis L. (Cannabaceae), hackberry. Map 127. BUFFALO County (2.2 mi on Hwy. S from Ft. Thompson toward Missouri River, $44^{\circ} 2.9^{\prime} \mathrm{N}$, $99^{\circ} 24.6^{\prime} \mathrm{W}$, Meyer \& Grassrope 1318) and HUGHES County (4 mi S on Rd. 21756 off Hwy. 34, at Missouri River, Meyer et al. 1518).

Chenopodium glaucum L. (Amaranthaceae), oakleaved goosefoot. Map 258. BUFFALO County (2.1 mi E on Hwy. 34 from Hwy. 47, $44^{\circ} 4.6^{\prime} \mathrm{N}$, $99^{\circ} 17.0^{\prime} \mathrm{W}$, Meyer 1357 ).

Chenopodium pratericola Rydb. (Amaranthaceae). Map 256. BUFFALO County $(0.5 \mathrm{mi} \mathrm{N}$ of 236 St. \& 349 Ave., behind the Whiting home, $43^{\circ} 58.0^{\prime} \mathrm{N}, 99^{\circ} 12.4^{\prime} \mathrm{W}$, Meyer et al. 1297).

Chenopodium simplex (Torr.) Raf. [Chenopodium hybridum L.] (Amaranthaceae), maple-leaved goosefoot. Map 259. This plant was called C. gigantospermum Aellen by Great Plains Flora Association (1991), which notes the American species to be properly separated from $C$. hybridum sensu stricto, a European taxon.
HUGHES County (2.6 mi off Hwy. 34 on Rd. $21756,44^{\circ} 14^{\prime} \mathrm{N}, 99^{\circ} 41^{\prime} \mathrm{W}$, Meyer et al. 1284) and BUFFALO County (1.5 mi off Hwy. 47 on road to E near Stephan, $44^{\circ} 13.0^{\prime} \mathrm{N}, 99^{\circ} 25.5^{\prime} \mathrm{W}$, Meyer et al. 1367).

Chorispora tenella (Pall.) DC. (Brassicaceae), blue mustard. Map 452. BUFFALO County (Crow Creek river bottom along SD Route $50,2 \mathrm{mi} \mathrm{S}$ of Route $47,43^{\circ} 58.7^{\prime} \mathrm{N}, 99^{\circ} 18.9^{\prime} \mathrm{W}$, Townesmith \& Applequist 2455).

Cirsium flodmanii (Rydb.) Arthur (Asteraceae), prairie thistle. Map 1430. BUFFALO County (1 mi N on Hwy. 47 from Ft. Thompson, then E 0.5-1 mi, $44^{\circ} 5.2^{\prime} \mathrm{N}, 9^{\circ} 25.7^{\prime} \mathrm{W}$, Meyer b Pemberton 1439).

Cirsium undulatum (Nutt.) Spreng. (Asteraceae), wavyleaf thistle. Map 1433. BUFFALO County (0.5 mi N of 236 St. \& 349 Ave., $43^{\circ} 58.0^{\prime} \mathrm{N}$, $99^{\circ} 12.4^{\prime} \mathrm{W}$, Meyer et al. 1293).

Dalea enneandra Nutt. (Fabaceae), nineanther dalea. Map 668. BUFFALO County $(2.5 \mathrm{mi} \mathrm{S}$ on Hwy. 47 from Hwy 50, 43⒌ $579^{\prime}$ N, 99 $18.4^{\prime} \mathrm{W}$, Meyer et al. 1577).

Delphinium carolinianum Walter subsp. virescens (Nutt.) R.E. Brooks [Delphinium virescens Nutt.] (Ranunculaceae), prairie larkspur. Map 85. BUFFALO County $(0.5 \mathrm{mi} \mathrm{N}$ of $236 \mathrm{St}$. \& 349 Ave., $43^{\circ} 58.0^{\prime} \mathrm{N}, 99^{\circ} 12.4^{\prime} \mathrm{W}$, Meyer et al. 1392 and Meyer et al. 1529).

Dichanthelium oligosanthes (Schult.) Gould var. scribnerianum (Nash) Gould [Panicum oligosanthes Schult. var. scribnerianum (Nash) Fernald] (Poaceae), small panicgrass. Map 2037. BUFFALO County $(0.5 \mathrm{mi} \mathrm{N}$ of $236 \mathrm{St}$. \& 349 Ave., $43^{\circ} 58.0^{\prime} \mathrm{N}, 9^{\circ} 12.4^{\prime} \mathrm{W}$, Meyer \& Pemberton 1251).

Drymocallis arguta (Pursh) Rydb. [Potentilla arguta Pursh] (Rosaceae), tall cinquefoil. Map 571. This species has been placed within Potentilla in most recent literature, but the forthcoming Flora of North America treatment of Potentilleae will recognize several segregate genera, including Drymocallis (Ertter 2007). BUFFALO County (0.5 mi N of 236 St. \& 349 Ave., $43^{\circ} 58.0^{\prime} \mathrm{N}, 99^{\circ} 12.4^{\prime} \mathrm{W}$, Meyer et al. 1563).

Drymocallis glandulosa (Lindl.) Rydb. [Potentilla glandulosa Lindl.] (Rosaceae), sticky cinquefoil. As traditionally defined, this is a mostly northwestern species whose range at the eastern extreme extends into western South Dakota, with a disjunct population reported in the eastern Codington County. Ertter (2007) implies that it may be considered strictly western in her "unabashedly provisional" treatment, but does not make clear how the most eastern populations will be otherwise classified. BUFFALO County (2.2 mi on Hwy. $47 \mathrm{~S}$ from Ft. Thompson toward Missouri River, $44^{\circ} 2.9^{\prime}$ N, $99^{\circ} 24.6^{\prime} \mathrm{W}$, Meyer \& Grassrope 1314). 
Echinochloa crus-galli (L.) Beauv. (Poaceae), barnyardgrass. Map 1949. BUFFALO County (1.5 mi off Hwy. 47 on road to E near Stephan, $44^{\circ} 13.0^{\prime} \mathrm{N}, 9^{\circ} 25.5^{\prime} \mathrm{W}$, Meyer et al. 1361).

Elaeagnus angustifolia L. (Elaeagnaceae), Russian olive. Map 809. HUGHES County (2.5 mi E from West Bend Rd./15 \& 320 Rd., $0.6 \mathrm{mi}$ on access road to Missouri River, $44^{\circ} 8.5^{\prime} \mathrm{N}$, $99^{\circ} 49.0^{\prime} \mathrm{W}, 5$ July 2012, Meyer et al. 1502 and Meyer et al. 1503).

Eleocharis erythropoda Steud. (Cyperaceae). Map 1826. HUGHES County (2.6 mi off Hwy. 34 on Rd. $21756,44^{\circ} 14^{\prime} \mathrm{N}, 99^{\circ} 41^{\prime} \mathrm{W}$, Meyer et al. 1466).

Elymus virginicus L. (Poaceae), Virginia wild rye. Map 1958. HUGHES County (S on Rd. 21756 from Hwy. 34, W on side road, at stock dam, $44^{\circ} 15.3^{\prime} \mathrm{N}, 9^{\circ} 41.6^{\prime} \mathrm{W}$, Meyer et al. 1277; 2.6 mi off Hwy. 34 on Rd. 21756, 44 $13.6^{\prime} \mathrm{N}$, $99^{\circ} 41.1^{\prime} \mathrm{W}$, Meyer et al. 1288).

Euphorbia marginata Pursh (Euphorbiaceae), snowon-the-mountain. HUGHES County $(2.2 \mathrm{mi}$ off Hwy. 34 on Rd. 21756, 44 $14^{\prime}$ N, $99^{\circ} 41^{\prime} \mathrm{W}$, Meyer et al. 1531; Joe Creek Recreation Area, $44^{\circ} 8.8^{\prime} \mathrm{N}, 99^{\circ} 47.8^{\prime} \mathrm{W}$, Meyer et al. 1566).

Euphorbia spathulata Lam. (Euphorbiaceae). Map 859. HUGHES County (2.6 mi off Hwy. 34 on Rd. $21756,44^{\circ} 14^{\prime} \mathrm{N}, 99^{\circ} 41^{\prime} \mathrm{W}$, Meyer et al. $1285)$ and BUFFALO County $(0.5 \mathrm{mi} \mathrm{N}$ of 236 St. \& 349 Ave., $43^{\circ} 58.0^{\prime} \mathrm{N}, 99^{\circ} 12.4^{\prime} \mathrm{W}$, Meyer et al. 1295 and Meyer et al. 1402).

Fraxinus pennsylvanica Marshall (Oleaceae), red or green ash. Maps 1193 and 1194. The species was collected from HUGHES County $(2.6 \mathrm{mi}$ off Hwy. 34 on Rd. 21756, 44 $13.6^{\prime} \mathrm{N}, 99^{\circ}$ 41.1' W, Meyer et al. 1468).

Galium boreale L. (Rubiaceae), northern bedstraw. Map 1290. HUGHES County (S from Hwy. 34 on Rd. 21756, W on side road, at stock dam, $44^{\circ} 15.3^{\prime} \mathrm{N}, 99^{\circ} 41.6^{\prime} \mathrm{W}$, Meyer \& Pemberton 1267).

Geranium viscosissimum Fisch. \& C.A. Mey. (Geraniaceae). This primarily Western species has been reported only from a few scattered localities in the Great Plains, including 3 of the westernmost counties in South Dakota. A collection from HUGHES County (S from Hwy. 34 on Rd. $21756, \mathrm{~W}$ on side road, at stock dam, $44^{\circ} 15.3^{\prime} \mathrm{N}, 99^{\circ} 41.6^{\prime} \mathrm{W}$, Meyer \& Pemberton 1422) represents a significant extension of its range.

Gleditsia triacanthos L. (Fabaceae), honey locust. HUGHES County (2.5 mi E from West Bend Rd./15 \& $320 \mathrm{Rd}$., $0.6 \mathrm{mi}$ on access road to Missouri River, $44^{\circ} 8.5^{\prime} \mathrm{N}, 99^{\circ} 49.0^{\prime} \mathrm{W}$, Meyer et al. 1498).

Grindelia squarrosa (Pursh) Dunal (Asteraceae), curlytop gumweed. Maps 1488-1490. HUGHES County $(1.3 \mathrm{mi}$ off West Bend Rd. on Rd. $320 / 15,44^{\circ} 9.6^{\prime} \mathrm{N}, 99^{\circ} 46.0^{\prime} \mathrm{W}$, Meyer et al.
1484; $2.6 \mathrm{mi}$ off Hwy. 34 on Rd. 21756, $44^{\circ} 13.6^{\prime} \mathrm{N}, 9^{\circ} 41.1^{\prime} \mathrm{W}$, Meyer et al. 1508; Big Bend District, near Pow-Wow grounds, Meyer et al. 1584).

Hedeoma hispida Pursh (Lamiaceae), rough pennyroyal. BUFFALO County $(0.5 \mathrm{mi} \mathrm{N}$ of 236 St. \& 349 Ave., $43^{\circ} 58.0^{\prime} \mathrm{N}, 99^{\circ} 12.4^{\prime} \mathrm{W}$, Meyer et al. 1482).

Helianthus pauciflorus Nutt. subsp. subrhomboideus (Rydb.) O. Spring \& E.E. Schill. [H. rigidus (Cass.) Desf. subsp. subrhomboideus (Rydb.) Heiser] (Asteraceae), stiff sunflower. Map 1515. BUFFALO County (Crow Creek District, 2.5 mi S of Hwy. 50 on Hwy. 47, 43 $58^{\prime} \mathrm{N}, 99^{\circ}$ $18^{\prime} \mathrm{W}$, Meyer et al. 1570).

Heliopsis helianthoides (L.) Sweet var. scabra (Dunal) Fernald (Asteraceae), false sunflower. Map 1519. HUGHES County (Rd. 21756, $2.6 \mathrm{mi} \mathrm{S}$ of Hwy. 34, 44 $13.6^{\prime} \mathrm{N}, 99^{\circ} 41.1^{\prime} \mathrm{W}$, Meyer et al. 1278 and Meyer et al. 1286).

Heterotheca villosa (Pursh) Shinners var. villosa [Chrysopsis villosa (Pursh.) Nutt. var. villosa] (Asteraceae), golden aster. Map 1417. HUGHES County (Joe Creek Recreation Area, $44^{\circ} 8.8^{\prime} \mathrm{N}$, $99^{\circ} 47.8^{\prime} \mathrm{W}$, Meyer 1349).

Hieracium umbellatum L. (Asteraceae). Map 1524. BUFFALO County $(0.5 \mathrm{mi} \mathrm{N}$ of $236 \mathrm{St}$. \& 349 Ave., $43^{\circ} 58.0^{\prime} \mathrm{N}, 99^{\circ} 12.4^{\prime} \mathrm{W}$, Meyer et al. 1301).

Juncus dudleyi Wiegand (Juncaceae), Dudley rush. Map 1715. HUGHES County (2.6 mi off Hwy. 34 on Rd. $21756,44^{\circ} 13.6 \mathrm{~N}, 99^{\circ} 41.1 \mathrm{~W}$, Meyer et al. 1291) and BUFFALO County (Old Ft. Thompson, near dam, along Missouri River, $44^{\circ} 12^{\prime} \mathrm{N}, 99^{\circ} 26^{\prime} \mathrm{W}$, Meyer et al. 1362).

Juncus nodosus L. (Juncaceae), knotted rush. Map 1721. HUGHES County (S from Hwy. 34 on Rd. 21756, W on side road, at stock dam, $44^{\circ} 15.3^{\prime} \mathrm{N}, 9^{\circ} 41.6^{\prime} \mathrm{W}$, Meyer \& Pemberton 1269; $2.6 \mathrm{mi}$ on Rd. 21756 off Hwy. 34, $44^{\circ} 13.6^{\prime} \mathrm{N}, 99^{\circ} 41.1^{\prime} \mathrm{W}$, Meyer et al. 1475).

Juniperus virginiana L. (Cupressaceae), red cedar. Map 52. HUGHES County (Joe Creek Recreation Area, $44^{\circ} 8.8^{\prime} \mathrm{N}, 99^{\circ} 47.8^{\prime} \mathrm{W}$, Meyer et al. 1569; toward North Shore Recreation Area on Rd. $21756,44^{\circ} 14.8^{\prime} \mathrm{N} 99^{\circ} 40.5^{\prime} \mathrm{W}$, Meyer 1258 and 1259).

Koeleria macrantha (Ledeb.) Schult. [Koeleria pyramidata (Lam.) Beauv.] (Poaceae), junegrass. Map 1990. This species has previously been synonymized with $K$. pyramidata, which, however, is a distinct European species (Arnow 1994). HUGHES County (2.6 mi on Rd. 21756 off Hwy. 34, $44^{\circ} 13.6^{\prime}$ N, $99^{\circ} 41.1^{\prime}$ W, Meyer et al. 1271 .

Lactuca serriola L. (Asteraceae), prickly lettuce. Map 1549. HUGHES County (2.6 mi on County Rd. 21756 toward North Shore Recreation Area, $44^{\circ} 14^{\prime} \mathrm{N}, 99^{\circ} 41^{\prime} \mathrm{W}$, Meyer et al. $1283)$ and BUFFALO County $(2.5 \mathrm{mi} \mathrm{S}$ on 
Hwy. 47 from Hwy. 50, 43⒌ $579^{\prime}$ N, $99^{\circ} 18.4^{\prime} \mathrm{W}$, Meyer et al. 1571).

Lomatium foeniculaceum (Nutt.) J.M. Coult. \& Rose var. foeniculaceum (Apiaceae), wild parsley. Map 945. HUGHES County (around North Bend Recreation Area, $44^{\circ} 13^{\prime} 34^{\prime \prime} \mathrm{N}, 99^{\circ} 41^{\prime}$ $06^{\prime \prime} \mathrm{W}$, Applequist \& Townesmith 321), BUFFALO County $(0.5 \mathrm{mi} \mathrm{N}$ of 236 th St. and 349th Ave.; $43^{\circ} 58.0^{\prime} \mathrm{N}, 99^{\circ} 12.4^{\prime} \mathrm{W}$, Applequist of Townesmith 324), and HYDE County (T109N R70W S25, 1 mi SE of intersection of SD Routes 47/34, W of road, Townesmith 2465).

Lomatium orientale J.M. Coult. \& Rose (Apiaceae), wild parsley. Map 948. HUGHES County (near stock dam W of Rd. 21756, 44 $15.3^{\prime} \mathrm{N}$, $99^{\circ} 41.6^{\prime} \mathrm{W}$, Applequist \& Townesmith 337) and BUFFALO County (along unimproved extension of 334th Ave., $3.0 \mathrm{mi} \mathrm{N}$ of $226 \mathrm{th}$ St., hillsides above Missouri River, $44^{\circ} 8.8^{\prime} \mathrm{N}, 99^{\circ}$ $31.2^{\prime} \mathrm{W}$, Townesmith 2469 and Townesmith 2470).

Lonicera tatarica L. (Caprifoliaceae), Tatarian honeysuckle. Map 1308. HUGHES County (Joe Creek Recreation Area, $44^{\circ} 8.8^{\prime} \mathrm{N}, 99^{\circ}$ 47.8' W, Meyer 1342).

Lycopus americanus Muhl. ex W.P.C. Barton (Lamiaceae), American bugleweed. BUFFALO County (2.1 mi E on Hwy. 34 from Hwy. 47, 44 4.6' $\mathrm{N}$, $99^{\circ} 17.0^{\prime} \mathrm{W}$, Meyer 1355$)$.

Lygodesmia juncea (Pursh) D. Don ex Hook. (Asteraceae), skeletonweed. Map 1559. BUFFALO County (2.1 mi on SD Rd. 8 from North Shore turnoff, shelterbelt, $44^{\circ} 5.5^{\prime} \mathrm{N}, 99^{\circ} 32.0^{\prime} \mathrm{W}$, Meyer 1359).

Maianthemum stellatum (L.) Link [Smilacina stellata (L.) Desf.] (Asparagaceae), spikenard. Map 2171. HUGHES County (S from Hwy. 34 on Rd. 21756, W on side road, at stock dam, $44^{\circ} 15.3^{\prime} \mathrm{N}, 9^{\circ} 41.6^{\prime} \mathrm{W}$, Meyer \& Pemberton 1421; same loc., Applequist \& Townesmith 340).

Medicago lupulina L. (Fabaceae), black medic. BUFFALO County $(0.5 \mathrm{mi} \mathrm{N}$ of $236 \mathrm{St}$. \& 349 Ave., $43^{\circ} 58.0^{\prime} \mathrm{N}, 99^{\circ} 12.4^{\prime} \mathrm{W}$, Meyer et al. 1393).

Medicago sativa L. (Fabaceae), alfalfa. Map 705. BUFFALO County $(0.5 \mathrm{mi} \mathrm{N}$ of $236 \mathrm{St}$. \& 349 Ave., $43^{\circ} 58.0^{\prime} \mathrm{N}, 99^{\circ} 12.4^{\prime} \mathrm{W}$, Meyer et al. 1559).

Melilotus albus Medik. (Fabaceae), white sweet clover. Map 706. BUFFALO County $(0.1 \mathrm{mi}$ $\mathrm{E}$ on Pomani Rd. from Eagle Rd., $44^{\circ} 4.6^{\prime} \mathrm{N}$, $99^{\circ} 27.6^{\prime} \mathrm{W}$, Meyer 1371) and HUGHES County (Big Bend District, $2.6 \mathrm{mi}$ on Rd. 21756 toward North Shore Recreation Area, Cheney Rush tributary, $44^{\circ} 14^{\prime} \mathrm{N}, 99^{\circ} 41^{\prime} \mathrm{W}$, Meyer 1255)

Melilotus officinalis (L.) Lam. (Fabaceae), yellow sweet clover. Map 707. BUFFALO County (2.8 mi S on Hwy. 47 from Hwy. 50, 43⒌ $57.9^{\prime} \mathrm{N}$, $99^{\circ} 18.4^{\prime} \mathrm{W}$, Meyer \& Claymore 1369; $0.5 \mathrm{mi} \mathrm{N}$ of 236 St. \& 349 Ave., $43^{\circ} 58.0^{\prime} \mathrm{N}, 99^{\circ} 12.4^{\prime} \mathrm{W}$, Meyer et al. 1562).

Mentha arvensis L. (Lamiaceae), field mint. Map 1147. HUGHES County (S from Hwy. 34 on Rd. 21756, W on side road, at stock dam, $44^{\circ} 15.3^{\prime} \mathrm{N}, 99^{\circ} 41.6^{\prime} \mathrm{W}$, Meyer et al. 1586) and BUFFALO County (2.1 mi E on Hwy. 34 from Hwy. 47, 44 $4.6^{\prime} \mathrm{N}, 99^{\circ} 17.0^{\prime} \mathrm{W}$, Meyer 1356 ).

Mentzelia nuda (Pursh) Torr. \& A. Gray (Loasaceae), bractless mentzelia. Map 392. HUGHES County (Joe Creek Recreation Area, $44^{\circ} 8.8^{\prime} \mathrm{N}$, $99^{\circ} 47.8^{\prime}$ W, Meyer 1340).

Monarda fistulosa L. (Lamiaceae), wild bergamot. Maps 1150 and 1151. HUGHES County (1.1 mi on SD County Rd. 21756, $0.4 \mathrm{mi}$ to ravine, $0.6 \mathrm{mi}$ to fence, $44^{\circ} 15.3^{\prime} \mathrm{N}, 99^{\circ} 41.6^{\prime} \mathrm{W}$, Meyer et al. 1456).

Musineon divaricatum (Pursh) Nutt. (Apiaceae). Map 949. BUFFALO County (Crow Creek river bottom along $\mathrm{SD}$ route $50,2 \mathrm{mi} \mathrm{S}$ of Route $47,43^{\circ} 58.7^{\prime} \mathrm{N}, 99^{\circ} 18.9^{\prime} \mathrm{W}$, Townesmith \& Applequist 2456; along unimproved extension of 334th Ave., $3.0 \mathrm{mi} \mathrm{N}$ of 226 th St., hillsides above Missouri River, $44^{\circ} 8.8^{\prime} \mathrm{N}, 99^{\circ} 31.2^{\prime}$ W, Townesmith 2468).

Oenothera villosa Thunb. (Onagraceae), hairy evening primrose. BUFFALO County $(2.2 \mathrm{mi}$ on Hwy. S of Ft. Thompson toward Missouri River, $44^{\circ} 2.9^{\prime} \mathrm{N}, 99^{\circ} 24.6^{\prime} \mathrm{W}$, Meyer \& Grassrope 1320 ).

Opuntia macrorhiza Engelm. (Cactaceae), bigroot prickly pear. Map 192. BUFFALO County (1 mi $\mathrm{N}$ on Hwy 47 from Ft. Thompson, then E 0.5-1 mi, $44^{\circ} 5.2^{\prime} \mathrm{N}, 99^{\circ} 25.7^{\prime} \mathrm{W}$, Meyer \& Pemberton 1449).

Oxalis dillenii Jacq. (Oxalidaceae). Map 901. BUFFALO County $(0.5 \mathrm{mi} \mathrm{N}$ of 236 St. \& 349 Ave., $43^{\circ} 58.0^{\prime} \mathrm{N}, 99^{\circ} 12.4^{\prime} \mathrm{W}$, Meyer \& Pemberton 1415).

Oxytropis lambertii Pursh (Fabaceae), purple locoweed. Map 709. BUFFALO County $(0.5 \mathrm{mi} \mathrm{N}$ of 236 St. \& 349 Ave., $43^{\circ} 58.0^{\prime} \mathrm{N}, 99^{\circ} 12.4^{\prime} \mathrm{W}$, Meyer et al. 1391).

Panicum virgatum L. (Poaceae), switchgrass. Map 2044. BUFFALO County (1.0 m S on Hwy. 47 from Hwy. 34, at bridge, $44^{\circ} 2.9^{\prime} \mathrm{N}, 99^{\circ} 24.6^{\prime} \mathrm{W}$, Meyer \& Grassrope 1329).

Parthenocissus vitacea (Knerr) Hitchc. (Vitaceae), woodbine. Map 873. HUGHES County (S from Hwy. 34 on Rd. 21756, W on side road, at stock dam, $44^{\circ} 15.3^{\prime} \mathrm{N}, 99^{\circ} 41.6^{\prime} \mathrm{W}$, Meyer \& Pemberton 1264).

Pascopyrum smithii (Rydb.) Barkworth \& D.R. Dewey [Agropyrum smithii Rydb.] (Poaceae), western wheatgrass. Map 1871. BUFFALO County (2.1 mi on SD Rd. 8 from North Shore turn off, $44^{\circ} 5.5^{\prime} \mathrm{N}, 99^{\circ} 32.0^{\prime} \mathrm{W}$, Meyer 1360 ; $1 \mathrm{mi} \mathrm{N}$ on Hwy. 47 from Ft. Thompson, then E 0.5-1 mi, $44^{\circ} 5.2^{\prime} \mathrm{N}, 99^{\circ} 25.7^{\prime} \mathrm{W}$, Meyer \& Pemberton 1440 . 
Phalaris arundinacea L. (Poaceae), reed canarygrass. Map 2054. HUGHES Co. (S from Hwy. 34 on Rd. $21756, \mathrm{~W}$ on side road, at stock dam, $44^{\circ} 15.3^{\prime} \mathrm{N}, 99^{\circ} 41.6^{\prime} \mathrm{W}$, Meyer \& Pemberton 1427).

Phragmites australis (Cav.) Trin. ex Steud. (Poaceae) subsp. americanus Saltonstall, P.M. Peterson \& Soreng, common reed. Map 2058. BUFFALO County $(0.1 \mathrm{mi} \mathrm{E}$ on Pomani Rd. from Eagle

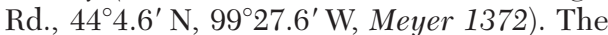
specimen is sterile, but the ligule length of about $1.4 \mathrm{~mm}$ suffices to indicate that it belongs to the native North American lineage rather than to the more common introduced lineage (Saltonstall et al. 2004).

Pinus ponderosa P. Lawson \& C. Lawson (Pinaceae), Ponderosa pine. Map 46. HUGHES County (Joe Creek Recreation Area, 44 $8.8^{\prime} \mathrm{N}, 99^{\circ}$ $47.8^{\prime} \mathrm{W}$, Meyer 1343). This specimen represents the northeasternmost known locality in South Dakota for this primarily western species.

Plantago major L. (Plantaginaceae), common plantain. Map 1184. HUGHES County $(2.6 \mathrm{mi}$ on County Rd. 21756 toward North Shore Recreation Area, $44^{\circ} 14^{\prime} \mathrm{N}, 99^{\circ} 41^{\prime} \mathrm{W}$, Meyer et al. 1276).

Poa pratensis L. (Poaceae), Kentucky bluegrass. Map 2071. BUFFALO County (0.5 mi N of 236 St. \& 349 Ave., $43^{\circ} 58.0^{\prime} \mathrm{N}, 99^{\circ} 12.4^{\prime} \mathrm{W}$, Meyer et al. 1245).

Polygala alba Nutt. (Polygalaceae), white milkwort. Map 918. BUFFALO County $(0.5 \mathrm{mi} \mathrm{N}$ of 236 St. \& 349 Ave., $43^{\circ} 58.0^{\prime} \mathrm{N}, 99^{\circ} 12.4^{\prime} \mathrm{W}$, Meyer et al. 1388).

Polygala verticillata L. (Polygalaceae), whorled milkwort. Map 922. HUGHES County (2.6 mi on County Rd. 21756 toward North Shore Recreation Area, Cheney Rush Creek, $44^{\circ} 14^{\prime} \mathrm{N}$, $99^{\circ} 41^{\prime} \mathrm{W}$, Meyer et al. 1287).

Potentilla paradoxa Nutt. (Rosaceae), bushy cinquefoil. Map 577. BUFFALO County (2.2 mi on Hwy. $47 \mathrm{~S}$ of Ft. Thompson toward Missouri River, $44^{\circ} 2.9^{\prime} \mathrm{N}, 99^{\circ} 24.6^{\prime} \mathrm{W}$, Meyer of Grassrope 1313).

Quercus macrocarpa Michx. (Fagaceae), bur oak. Map 156. HUGHES County (2.6 mi S of Hwy. 34 on SD Rd. $21756,44^{\circ} 13.6^{\prime} \mathrm{N}, 99^{\circ} 41.1^{\prime} \mathrm{W}$, Meyer et al. 1469).

Ranunculus cymbalaria Pursh (Ranunculaceae), shore buttercup. Map 91. HUGHES County (2.6 mi S of Hwy. 34 on SD Rd. 21756, Cheney Rush Creek, $44^{\circ} 13.6^{\prime} \mathrm{N}, 99^{\circ} 41.1^{\prime} \mathrm{W}$, Meyer et al. 1290).

Rhus glabra L. (Anacardiaceae), smooth sumac. Map 892. HUGHES County (Joe Creek Recreation Area, $44^{\circ} 8.8^{\prime} \mathrm{N}, 99^{\circ} 47.8^{\prime} \mathrm{W}$, Meyer 1347).

Rorippa curvipes Greene (Brassicaceae). BUFFALO County (2.2 mi on Hwy. $47 \mathrm{~S}$ of Ft. Thompson toward Missouri River, $44^{\circ} 2.9^{\prime} \mathrm{N}, 99^{\circ} 24.6^{\prime} \mathrm{W}$, Meyer \& Grassrope 1322).
Rumex maritimus L. [Rumex maritimus L. var. fueginus (Phil.) Dusen] (Polygonaceae), golden dock. Map 330. BUFFALO County $(2.0 \mathrm{mi}$ on Hwy. $47 \mathrm{~S}$ of Hwy. 34, at Rd. 235, Crow

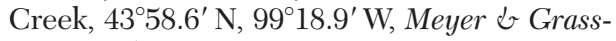
rope 1339).

Salix amygdaloides Andersson (Salicaceae), peachleaved willow. Map 406. BUFFALO County (2.2 mi on Hwy. $47 \mathrm{~S}$ of Ft. Thompson toward Missouri River, $44^{\circ} 2.9^{\prime} \mathrm{N}, 9^{\circ} 24.6^{\prime} \mathrm{W}$, Meyer \& Grassrope 1324; $2.0 \mathrm{mi}$ on Hwy. $47 \mathrm{~S}$ of Hwy. 34, at Rd. 235, Crow Creek, 43558.6’ N, $99^{\circ} 18.9^{\prime} \mathrm{W}$, Meyer \& Grassrope 1338; $2.1 \mathrm{mi} \mathrm{E}$

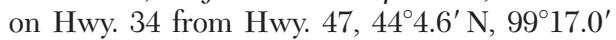
W, Meyer 1354; $2.2 \mathrm{mi} \mathrm{S}$ of Ft. Thompson on Hwy. 47, ravine to $\mathrm{S}$ of road, $44^{\circ} 2.9^{\prime} \mathrm{N}, 99^{\circ}$ 24.6' W, Applequist \& Townesmith 330).

Salix interior Rowlee [Salix exigua Nutt. subsp. interior (Rowlee) Cronquist] (Salicaceae), coyote willow. Map 412. The most recent treatment of North American Salix (Argus 2010) recognizes $S$. interior as distinct from the widespread western species $S$. exigua, though distinguishing characters, such as flat vs. minutely revolute leaf margins, are small, and the two are known to hybridize. BUFFALO County (Crow Creek river bottom along SD Route $50,2 \mathrm{mi} \mathrm{S}$ of Route $47,43^{\circ} 58.7^{\prime} \mathrm{N}$, $99^{\circ} 18.9^{\prime} \mathrm{W}$, Townesmith \& Applequist 2454).

Schizachyrium scoparium (Michx.) Nash [Andropogon scoparius Michx.] (Poaceae), little bluestem. Map 1888. BUFFALO County (2.8 mi S

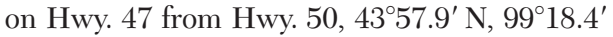
W, Meyer \& Claymore 1368).

Scirpus pallidus (Britt.) Fern. [Scirpus atrovirens Willd. var. pallidus Britt.] (Cyperaceae), darkgreen bulrush. Map 1849. HUGHES County (S from Hwy. 34 on Rd. 21756, W on side road, at stock dam, $44^{\circ} 15.3^{\prime} \mathrm{N}, 99^{\circ} 41.6^{\prime} \mathrm{W}$, Meyer o Pemberton 1270).

Scrophularia lanceolata Pursh (Scrophulariaceae), figwort. Map 1245. HUGHES County (S from Hwy. 34 on Rd. 21756, W on side road, at stock dam, $44^{\circ} 15.3^{\prime} \mathrm{N}, 99^{\circ} 41.6^{\prime} \mathrm{W}$, Meyer \& Pemberton 1431).

Shepherdia argentea (Pursh) Nutt. (Elaeagnaceae), buffaloberry. Map 811. HUGHES County (S from Hwy. 34 on Rd. 21756, W on side road, at stock dam, $44^{\circ} 15.3^{\prime} \mathrm{N}, 99^{\circ} 41.6^{\prime} \mathrm{W}$, Meyer et al. 1266).

Silene antirrhina L. (Caryophyllaceae), sleepy catchfly. Map 221. BUFFALO County (0.5 mi N of 236 St. \& 349 Ave., $43^{\circ} 58.0^{\prime} \mathrm{N}, 99^{\circ} 12.4^{\prime} \mathrm{W}$, Meyer \& Pemberton 1418).

Solanum rostratum Dunal (Solanaceae), buffalo bur. Map 1030. HUGHES County (2.2 mi off Hwy. 34 on Rd. $21756,44^{\circ} 14^{\prime} \mathrm{N}, 99^{\circ} 41^{\prime} \mathrm{W}$, Meyer et al. 1536).

Solidago gigantea Aiton (Asteraceae), late goldenrod. Map 1616. BUFFALO County (2.6 mi on 
Rd. 232 off Hwy. 50, 44 $1.1^{\prime} \mathrm{N}, 99^{\circ} 17.0^{\prime} \mathrm{W}$, Meyer et al. 1579).

Solidago mollis Bartl. (Asteraceae), soft goldenrod. Map 1622. BUFFALO County $(0.5 \mathrm{mi} \mathrm{N}$ of 236 St. \& 349 Ave., $43^{\circ} 58.0^{\prime} \mathrm{N}, 99^{\circ} 12.4^{\prime} \mathrm{W}$, Meyer et al. 1376).

Solidago rigida L. var. humilis Porter (Asteraceae), rigid goldenrod. Map 1625. HUGHES County (S from Hwy. 34 on Rd. 21756, W on side road, at stock dam, $44^{\circ} 15.3^{\prime} \mathrm{N}, 99^{\circ} 41.6^{\prime} \mathrm{W}$, Meyer et al. 1379).

Sphaeralcea coccinea (Nutt.) Rydb. (Malvaceae), scarlet globemallow. Map 366. HUGHES County (2.2 mi off Hwy. 34 on Rd. 21756, $44^{\circ} 14^{\prime} \mathrm{N}, 99^{\circ} 41^{\prime} \mathrm{W}$, Meyer et al. 1532).

Strophostyles helvola (L.) Elliott (Fabaceae), wild bean. Map 732. This primarily eastern species is found mostly in the southern two-thirds of the eastern half of the Great Plains. A recent collection from HUGHES County $(2.5 \mathrm{mi} \mathrm{E}$ from the West Bend Rd./15 \& 320 Rd. intersection, $0.6 \mathrm{mi}$ on access road to Missouri River, $44^{\circ} 8.5^{\prime} \mathrm{N}, 99^{\circ} 49.0^{\prime} \mathrm{W}$, Meyer et al. 1504 ) represents a northwestward expansion of its known range.

Taraxacum officinale F.H. Wigg. (Asteraceae), dandelion. Map 1635. HUGHES County (around North Bend Recreation Area, 44 $13^{\prime} 34^{\prime \prime} \mathrm{N}$, $99^{\circ} 41^{\prime} 06^{\prime \prime} \mathrm{W}$, Applequist \& Townesmith 320).

Tradescantia bracteata Small (Commelinaceae), spiderwort. Map 426. HUGHES County (2.6 mi S on SD Rd. $21756,44^{\circ} 13.6^{\prime} \mathrm{N}, 99^{\circ} 41.1^{\prime} \mathrm{W}$, Meyer et al. 1465).

Tragopogon dubius Scop. (Asteraceae), goatsbeard. Map 1643. BUFFALO County (334th Ave. off 26th Ave., $1.5 \mathrm{mi}$ past Log House, $44^{\circ} 8.8^{\prime} \mathrm{N}$, $99^{\circ} 31.2^{\prime}$ W, Meyer et al. 1525).

Triodanis leptocarpa (Nutt.) Nieuw. (Campanulaceae). Map 1285. BUFFALO County $(0.5 \mathrm{mi}$ $\mathrm{N}$ of 236 St. \& 349 Ave., $0.5 \mathrm{mi}$ past Dewey Blvd., $43^{\circ} 58^{\prime} \mathrm{N}, 99^{\circ} 12^{\prime} \mathrm{W}$, Meyer et al. 1401).

Triodanis perfoliata (L.) Nieuw. (Campanulaceae). Map 1286. BUFFALO County $(0.5 \mathrm{mi} \mathrm{N}$ of 236 St. \& 349 Ave., 0.5 mi past Dewey Blvd., $43^{\circ} 58^{\prime} \mathrm{N}, 9^{\circ} 12^{\prime} \mathrm{W}$, Meyer b Pemberton 1419).

Ulmus americana L. (Ulmaceae), American elm. Map 130. HUGHES County (S from Hwy. 34 on Rd. $21756, \mathrm{~W}$ on side road, at stock dam, $44^{\circ}$ $15.3^{\prime} \mathrm{N}, 99^{\circ} 41.6^{\prime} \mathrm{W}$, Meyer et al. 1279; $2.5 \mathrm{mi} \mathrm{E}$ from the West Bend Rd./15 \& 320 Rd. intersection, $0.6 \mathrm{mi}$ on access road to Missouri River, $44^{\circ} 8.5^{\prime} \mathrm{N}, 99^{\circ} 49.0^{\prime} \mathrm{W}$, Meyer et al. 1500; around North Bend Recreation Area, $44^{\circ} 13^{\prime} 34^{\prime \prime} \mathrm{N}, 99^{\circ}$ 41'06" W, Applequist \& Townesmith 331).

Ulmus pumila L. (Ulmaceae), Siberian elm. Map 131. HUGHES County (1.3 mi on Rd. 320/15 off West Bend Rd., $44^{\circ} 9.6^{\prime} \mathrm{N}, 99^{\circ} 46.0^{\prime} \mathrm{W}$, Meyer et al. 1311; Joe Creek Recreation Area, $44^{\circ} 8.7^{\prime} \mathrm{N}, 99^{\circ} 47.8^{\prime} \mathrm{W}$, Townesmith \& Applequist 2461).
Verbena hastata L. (Verbenaceae), blue vervain. Map 1122. HUGHES County (1.1 mi down $\mathrm{SD} \mathrm{Rd} .21756,0.4 \mathrm{mi}$ to ravine, $0.6 \mathrm{mi}$ to fence, $44^{\circ} 15.3 \mathrm{~N}, 99^{\circ} 41.6 \mathrm{~W}$, Meyer et al. 1460 ; Joe Creek Recreation Area, $44^{\circ} 8.8^{\prime} \mathrm{N}, 99^{\circ} 47.8^{\prime} \mathrm{W}$, Meyer et al. 1567).

Vernonia fasciculata Michx. var. corymbosa (Schwein.) Schub. (Asteraceae), ironweed. Map 1653. BUFFALO County (0.5 mi N of 236 St. \& 349 Ave., $43^{\circ} 58.0^{\prime} \mathrm{N}, 99^{\circ} 12.4^{\prime} \mathrm{W}$, Meyer et al. 1296 and Meyer et al. 1375).

Vicia americana Muhl. ex Willd. (Fabaceae), American vetch. Maps 743 and 744. HUGHES County (2.2 mi off Hwy. 34 on Rd. 21756, 44 $14^{\prime} \mathrm{N}$, $99^{\circ} 41^{\prime} \mathrm{W}$, Meyer et al. 1405).

Viola adunca Sm. (Violaceae), small blue violet. Map 369. HUGHES County (near stock dam W of Rd. $21756,44^{\circ} 15.3^{\prime} \mathrm{N}, 99^{\circ} 41.6^{\prime} \mathrm{W}$, Applequist \& Townesmith 339).

Viola nuttallii Pursh (Violaceae), yellow prairie violet. Map 373. HUGHES County (around North Bend Recreation Area, 44 $13^{\prime} 34^{\prime \prime} \mathrm{N}$, 9941' 06" W, Applequist \& Townesmith 322)

Xanthium strumarium L. (Asteraceae), cocklebur. Map 1658. HUGHES County (1.1 mi down SD Rd. 21756, $0.4 \mathrm{mi}$ to ravine, $0.6 \mathrm{mi}$ to fence, $44^{\circ} 15.3^{\prime} \mathrm{N}, 99^{\circ} 41.6^{\prime} \mathrm{W}$, Meyer et al. 1453).

\section{North Dakota County Records}

Artemisia campestris L. subsp. caudata (Michx.) Hall \& Clem. (Asteraceae), western sagebrush. Map 1346. SIOUX County (N of Ft. Yates, ND, Prairie Knights Marina to the right, off walking trail near $\mathrm{W}$ parking lot, $46^{\circ} 15.9^{\prime} \mathrm{N}$, $100^{\circ} 34.9^{\prime} \mathrm{W}$, Meyer et al. 1544).

Liatris punctata Hook. (Asteraceae), blazing star. Map 1557. SIOUX County (hill behind Sitting Bull College, near wind turbine, $46^{\circ} 15.9^{\prime} \mathrm{N}$, $100^{\circ} 34.9^{\prime}$ W, Meyer d Claymore 1551).

\section{ACKNOWLEDGMENTS}

We thank the National Geographic Society for providing financial support for this research though grant number 8988-11; the Crow Creek Tribal Council for permitting field work; the Crow Creek High School for facilitating student participation; Peter Lengkeek and Linda Different Cloud Jones for facilitating fieldwork; George Yatskievych and John Pruski for assisting with specimen identification; and James Harris and an anonymous reviewer for helpful comments.

\section{Literature Cited}

Angiosperm Phylogeny Group. 2009. An update of the Angiosperm Phylogeny Group classification for 
the orders and families of flowering plants: APG III. Botanical Journal of the Linnaean Society 161: $105-121$

Argus, G.W. 2010. Salix. Pages 23-162 in Flora of North America Editorial Committee, editors, Flora of North America. Volume 7. Oxford University Press, New York, NY.

ARNOW, L.A. 1994. Koeleria macrantha and K. pyramidata (Poaceae): nomenclatural problems and biological distinctions. Systematic Botany 19:6-20.

Card, C., G. Kostel, and M. Gabel. 2010. A comprehensive herbarium database of vascular plants from northeastern Wyoming and western South Dakota. Proceedings of the South Dakota Academy of Sciences 89:77-83.

Ertter, B. 2007. Generic realignments in tribe Potentilleae and revision of Drymocallis (Rosoideae: Rosaceae) in North America. Journal of the Botanical Research Institute of Texas 1:31-46.

Great Plains Flora Association. 1977. Atlas of the Flora of the Great Plains. Iowa State University Press, Ames, IA
1991. Flora of the Great Plains. University Press of Kansas, Lawrence, KS.

Kartesz, J.T. 2013. The Biota of North America Program. North American Plant Atlas. [Accessed 31 Mar 2014]. Available from: http://bonap.net/napa

Rolfsmeier, S.B., R.B. Kaul, M.M. Garabrandt, and D.M. Sutherland. 1988. New and corrected floristic records for Nebraska. Transactions of the Nebraska Academy of Sciences 16:115-121.

Saltonstall, K., P.M. Peterson, and R.J. Soreng. 2004. Recognition of Phragmites australis subsp. americanus in North America: evidence from morphological and genetic analyses. Sida, Contributions to Botany 21:683-692.

USDA-NRCS. 2013. The PLANTS Database [online]. National Plant Data Team, USDA Natural Resources Conservation Service, Greensboro, NC; [accessed 15 November 2013]. Available from: http://plants.usda.gov

Received 31 December 2013 Accepted 18 August 2014 